\title{
Coronary Artery Calcification Compared with Carotid Intima- Media Thickness in Prediction of Cardiovascular Disease Incidence: The Multi-Ethnic Study of Atherosclerosis (MESA)
}

\author{
Aaron R. Folsom, MD ${ }^{1}$, Richard A. Kronmal, $\mathrm{PhD}^{2}$, Robert C. Detrano, MD, $\mathrm{PhD}^{3}$, Daniel H. \\ O'Leary, MD $^{4}$, Diane E. Bild, MD ${ }^{5}$, David A. Bluemke, MD, $\mathrm{PhD}^{6}$, Matthew J. Budoff, MD \\ Kiang Liu, PhD ${ }^{8}$, Steven Shea, MD ${ }^{9}$, Moyses Szklo, MD, DrPH ${ }^{10}$, Russell P. Tracy, PhD ${ }^{11}$, \\ Karol E. Watson, MD, PhD ${ }^{12}$, and Gregory L. Burke, MD ${ }^{13}$ \\ 1 Division of Epidemiology \& Community Health, School of Public Health, University of Minnesota, \\ Minneapolis, $M N$ \\ 2 Collaborative Health Studies Coordinating Center, University of Washington, Seattle, WA \\ 3 Department of Radiological Sciences, University of California at Irvine, Irvine, CA \\ 4 Caritas Carney Hospital, Dorchester, MA \\ 5 Division of Prevention \& Population Sciences, National Heart, Lung, and Blood Institute, Bethesda, MD \\ 6 Department of Radiology, Johns Hopkins University, School of Medicine, Baltimore, MD \\ 7 Division of Cardiology, Los Angeles Biomedical Research Institute at Harbor-UCLA Medical Center, \\ Torrance, $C A$
}

\footnotetext{
Corresponding Author: Aaron R. Folsom, MD, Division of Epidemiology \& Community Health, School of Public Health, University of Minnesota, Suite 300, 1300 South Second Street, Minneapolis, Minnesota 55454-1015, USA. Telephone: 612-626-8862. FAX: 612-624-0315. (folsom@epi.umn.edu).

Author Contributions: Dr. Folsom had full access to all of the data in the study and takes responsibility for the integrity of the data and the accuracy of the data analysis.

Study concept and design: Folsom, Kronmal, Detrano, O’Leary, Bild, Bluemke, Budoff, Burke, Liu, Shea, Szklo, Tracy, Watson. Acquisition of data: Folsom, Kronmal, Detrano, O’Leary, Bild, Bluemke, Budoff, Burke, Liu, Shea, Szklo, Tracy, Watson. Analysis and interpretation of data: Folsom, Kronmal.

Drafting of the manuscript: Folsom.

Statistical analysis: Kronmal.

Critical review of manuscript: Kronmal, Detrano, O’Leary, Bild, Bluemke, Budoff, Burke, Liu, Shea, Szklo, Tracy, Watson.

Financial Disclosures/Conflicts of Interest:

Dr. Folsom has no conflicts.

Dr. Kronmal has no conflicts.

Dr. Detrano has no conflicts.

Dr. O’Leary serves as consultant to Sankyo Pharma, Sanofi-Aventis, GlaxoSmithKline, Eli Lilly, Schering-Plough, Pfizer, AstraZeneca, and Merck. He also owns stock in Medpace.

Dr. Bild has no conflicts.

Dr. Bluemke has no conflicts.

Dr. Budoff has received honoraria for speaking on behalf of General Electric.

Dr. Burke has no conflicts.

Dr. Liu has no conflicts.

Dr. Shea has no conflicts.

Dr. Szklo has no conflicts.

Dr. Tracy has no conflicts.

Dr. Watson has no conflicts.

Role of the Sponsor: National Heart, Lung, and Blood Institute staff had input into the design and conduct of this study; collection, management, analysis, and interpretation of the data; and in preparation of the manuscript.
} 
8 Department of Preventive Medicine, Northwestern University Medical School, Chicago, IL

9 Departments of Medicine and Epidemiology, Columbia University, New York City, NY

10 Department of Epidemiology, Johns Hopkins University, Baltimore, MD

11 Departments of Pathology and Biochemistry, University of Vermont, Colchester, VT

12 Division of Cardiology, UCLA School of Medicine, Los Angeles, CA

13 Division of Public Health Sciences, Wake Forest University Health Sciences, Winston-Salem, NC

\section{Abstract}

Context-Coronary artery calcium (CAC) and carotid intima-media thickness (IMT) are noninvasive measures of atherosclerosis that consensus panels have recommended as possible additions to risk factor assessment for predicting the probability of cardiovascular disease (CVD) occurrence.

Objective-To assess whether maximum carotid IMT or CAC (Agatston Score) is the better predictor of incident CVD.

Design, Setting, Patients-Prospective cohort study of 45-84 year-olds initially free of CVD $(n=6,698)$ in four ethnic groups, with standardized carotid IMT and CAC measures at baseline, in six field centers of the Multi-Ethnic Study of Atherosclerosis (MESA).

Main Outcome Measure(s)-Incident CVD events (coronary heart disease, stroke, and fatal CVD) over a maximum of 5.3 years of follow-up.

Results-There were $222 \mathrm{CVD}$ events during follow-up. CAC was associated more strongly than carotid IMT with risk of incident CVD. After adjustment for each other and traditional CVD risk factors, the hazard of CVD increased 2.1-fold (95\% CI 1.8-2.5) for each standard deviation greater level of log-transformed CAC, versus 1.3-fold (95\% CI 1.1-1.4) for each standard deviation greater maximum IMT. For coronary heart disease, the hazard ratios per standard deviation increment were 2.5-fold (95\% CI 2.1-3.1) for CAC and 1.2-fold (95\% CI 1.0-1.4) for IMT. An ROC analysis also suggested that CAC predicted incident CVD better than IMT did.

Conclusions-Although whether and how to clinically use bio-imaging tests of subclinical atherosclerosis remains a topic of debate, this study found that CAC predicts subsequent CVD events better than does carotid IMT.

Prospective epidemiologic studies have consistently documented that noninvasive measures of atherosclerosis, such as coronary artery calcium (CAC) and carotid intima-media thickness (IMT), are associated positively and strongly with future incidence of cardiovascular disease (CVD). For example, a meta analysis recently identified relative risks of coronary heart disease (CHD) of 1.0, 1.9, 4.3, 7.2, and 10.8 for CAC values of 0, 1-112, 100-400, 400-999, and $\geq 1000$, respectively. ${ }^{1}$ Another meta analysis reported significant relative risks of CHD of 1.26 for myocardial infarction and 1.32 for stroke for each 1-standard deviation increment of common carotid artery IMT. ${ }^{2}$

Whether and how to use these screening tests in clinical practice remains a matter of debate. Some task forces have recommended bio-imaging tests for atherosclerosis be considered for patients at intermediate risk of CHD (10-20\% risk in 10 years), for whom preventive interventions are often uncertain. 1,3 Another group recommended measuring CAC or IMT in all asymptomatic 45-75 year old men and 55-75 year old women, as a guide to clinical decision-making. 4 
CAC and IMT are only moderately correlated within individuals, ${ }^{5-10}$ so each test has some potential to be useful clinically to predict future CVD. Terry et al reported that CAC was associated more strongly than IMT with prevalent coronary artery stenosis. ${ }^{11}$ Brook et al confirmed this, but found that an estimate of carotid plaque area predicted coronary stenosis somewhat more strongly than even CAC. ${ }^{12}$ Neither of these cross-sectional studies assessed CVD incidence. The prospective Rotterdam Study found carotid plaques, increased IMT, aortic calcium, and low ankle-brachial blood pressure index predicted incident myocardial infarction fairly comparably, and the more subclinical measures present, the greater the risk. ${ }^{13}$ Their study did not examine CAC. Very recently, the first prospective study assessed the potential utility of measuring CAC versus IMT for global CVD risk prediction. Newman et al ${ }^{\mathrm{REF}}$ found that CAC and common carotid IMT similarly predicted CVD and CHD in adults 70 years of age and older, but IMT was the better predictor of stroke. We now also address this question prospectively in the Multi-Ethnic Study of Atherosclerosis (MESA).

\section{METHODS}

\section{MESA Cohort and Risk Factor Assessments}

MESA recruited 6,814 adults aged 45-84 from the populations near six Field Centers Baltimore, MD; Chicago, IL; Forsyth County, NC; Los Angeles, CA; New York, NY; and St. Paul, MN - to a baseline examination between July 2000 and September 2002. ${ }^{14}$ They were Caucasians (38\%), African Americans (28\%), Hispanics (22\%), and Chinese Americans (12\%) free of clinically recognized CVD drawn from households in geographically-defined areas (5 centers) or in an occupational union (New York). MESA conducted three subsequent examinations of the cohort between 2002 and 2007. Institutional Review Boards at each site approved the study, and all participants gave written informed consent.

Centrally-trained clinical teams collected information on cardiovascular risk factors during the baseline examination. They measured resting blood pressure three times in seated participants with a Dinamap model Pro 1000 automated oscillometric sphygmomanometer (Critikon, Tampa, FL). A central laboratory measured total and HDL-cholesterol and glucose levels from blood samples obtained after a 12 -hour fast. We defined diabetes as fasting glucose $\geq 6.99$ $\mathrm{mmol} / \mathrm{L}(126 \mathrm{mg} / \mathrm{dL})$ or use of hypoglycemic medication.

\section{CAC Assessment}

Scanning centers assessed CAC by chest-computed tomography using either a cardiac-gated electron-beam computed tomography scanner (Chicago, Los Angeles, and New York Field Centers) or a multi-detector computed tomography system (Baltimore, Forsyth County and St. Paul Field Centers). Certified technologists scanned all participants twice. A phantom of known physical calcium concentration was included in the field of view. A radiologist or cardiologist read all CT scans at a central reading center (Los Angeles Biomedical Research Institute at Harbor-UCLA in Torrance, CA) using an interactive scoring system similar to that of Yaghoubi et al. ${ }^{15}$ The reader-work station interface identified and quantified CAC from images calibrated according to the readings of the calcium phantom. The Agatston score, ${ }^{16}$ which is a pseudo-continuous variable derived from plaque densities and their areas in all coronaries, was computed. We used the average phantom-adjusted Agatston score for the two scans in all analyses. Carr et al have reported the details of the MESA computed tomography scanning and interpretation methods. ${ }^{17}$ Each participant and his/her physicians were notified whether the CAC scores, for the participant's age, were less than average, average, or greater than average. No recommendation was made about treatment. 


\section{Carotid IMT Assessment}

Trained technicians in each Field Center performed B-mode ultrasonography of the right and left near and far walls of the internal carotid and common carotid arteries. ${ }^{18}$ They used the Logiq 700 ultrasound device (General Electric Medical Systems, Waukesha, WI) to record images. An ultrasound reading center (Department of Radiology, New England Medical Center) measured maximal IMT of the internal and common carotid sites as the mean of the maximum IMT of the near and far walls of the right and left sides. In addition, for this paper, we created a composite $\mathrm{Z}$ score for overall maximal IMT by summing the two carotid IMT sites (if both were measured) after standardization (subtraction of the mean and division by the standard deviation of each measure), and then dividing by the standard deviation of the sum. If only one of the two measures was available, it was used. The resulting variable, hereafter referred to as Z score maximum IMT, has a mean of zero and a standard deviation of one. Each participant and his/her physicians were notified whether or not an accompanying Doppler assessment suggested significant carotid stenosis ( $\geq 50 \%)$, but no recommendation was made about treatment.

\section{CVD Follow-Up}

We followed the cohort for incident CVD events for a median of 3.9 years (max 5.3). At intervals of 9-12 months, a telephone interviewer contacted each participant to inquire about interim hospital admissions, cardiovascular outpatient diagnoses, and deaths. In order to verify self-reported diagnoses, we requested copies of all death certificates and medical records for hospitalizations and outpatient cardiovascular diagnoses. We also conducted next-of-kin interviews for out of hospital cardiovascular deaths. We obtained records on an estimated $98 \%$ of reported hospitalized cardiovascular events and some information on $95 \%$ of reported outpatient diagnostic encounters.

Two physicians, blinded to the CAC and IMT data, independently reviewed and classified CVD events and assigned incidence dates. If, after review and adjudication, disagreements persisted, a full mortality and morbidity review committee made the final classification. MESA criteria for events were adopted from the Atherosclerosis Risk in Communities Study, the Cardiovascular Health Study, and the Women's Health Initiative. Reviewers classified myocardial infarction as definite, probable or absent, based primarily on combinations of symptoms, ECG, and levels of cardiac biomarker (generally, troponins or creatine kinase myocardial band). Reviewers graded angina based on their clinical judgment as definite, probable or absent. Probable angina required symptoms of ischemia, as well as documentation that a physician had diagnosed and treated for angina. Definite angina also required objective diagnostic evidence of CHD. In this paper, we only included definite angina $(n=76)$, plus probable angina when accompanied by coronary revascularization $(n=5)$. The reviewers classified CHD or CVD death as present or absent based on hospital records and interviews with families. Definite fatal CHD required an MI within 28 days of death, chest pain within the 72 hours before death, or a history of CHD and the absence of a known non-atherosclerotic or non-cardiac cause of death. Neurologists reviewed and classified stroke as present if there was a focal neurologic deficit lasting 24 hours or until death, with a clinically relevant lesion on brain imaging, and no nonvascular cause.

For this report, we defined incident CVD as CHD (definite and probable myocardial infarction, definite coronary heart disease death, resuscitated cardiac arrest, definite angina, and probable angina associated with coronary revascularization), stroke (fatal or nonfatal), or other atherosclerotic CVD death. Follow-up went from the baseline examination until the first CVD event, loss to follow-up, death, or else through January 12, 2005. 


\section{Statistical Analysis}

From the 6,814 MESA participants, we excluded 77 missing both of the carotid IMT measures, 5 discovered to have had CVD events prior to baseline, and 34 with no follow-up data, leaving 6,698 for analysis. For most analyses, we either (1) categorized carotid IMT and CAC into three groups (the bottom $50 \%$ and the two upper quartiles), to accommodate the fact that $50 \%$ of participants had zero CAC, or (2) treated IMT and the natural logarithm (ln) of $(\mathrm{CAC}+1)$ as continuous variables. The $\ln (\mathrm{CAC}+1)$ transformation better normalized the $\mathrm{CAC}$ distribution. We used Cox proportional hazard regression to estimate hazard ratios. We performed tests for non-proportional hazards using Shoenfeld residuals; all were nonsignificant. Covariates for multivariable models included age (continuous), sex, race/ethnicity (4 groups), smoking (current, former, never), diabetes (yes, no), blood pressure (6 categories per JNC 6, including medications), ${ }^{19} \mathrm{HDL}$ and total cholesterol (continuous), and use of lipid lowering medication (yes, no). We compared the strength of association for IMT versus CAC based on the relative size of their hazard ratios and the corresponding $\mathrm{X}^{2}$ test or $\mathrm{Z}$ test of the hazard ratios. We also compared IMT and CAC associations with receiver-operating characteristic curves (ROC) modeling carotid IMT and $\ln (\mathrm{CAC}+1)$ as a continuous variables in Cox models. Rates for Figure 1 were calculated for "low, medium, and high" values of $Z$ score maximum IMT and CAC using intervals as described above. All analyses were done using STATA 9.2 (StataCorp, College Station, Texas).

\section{RESULTS}

The MESA sample for this analysis comprised 6,698 adults age 45-84 years at baseline (3,161 men, 3,537 women). Over 23,735 person-years of follow-up, we identified incident 222 CVD events (159 CHD [61 myocardial infarction, 81 angina, 3 resuscitated cardiac arrest, 13 CHD deaths]; 59 stroke, of whom 3 also had a CHD event; and 7 other atherosclerotic CVD deaths). Fifty percent of the MESA sample had detectable CAC. The mean \pm SD value for $\ln (\mathrm{CAC} \pm 1)$ was $2.2 \pm 2.5$, for maximum internal carotid IMT was $1.07 \pm 0.60 \mathrm{~mm}$, for maximum common carotid IMT was $0.87 \pm 0.19 \mathrm{~mm}$ and for $\mathrm{Z}$ score maximum IMT was $0.00 \pm 1.00$.

As shown in Table 1, the three measures of carotid IMT were all positively associated with incident CVD, with age, race/ethnicity, and sex-adjusted HRs for the highest versus lowest quartile of 3.3 (95\% CI 2.1-5.2) for the maximum internal carotid IMT, 2.3 (95\% CI 1.4-3.8) for the maximum common carotid IMT, and 3.8 (95\% CI 2.2-6.4) for the Z score maximum IMT (all $\mathrm{p}<0.0001$ ). The remaining IMT analyses therefore focused on $\mathrm{Z}$ score maximum IMT. For CAC (Table 1), the HRs of CVD increased across categories, the age, race/ethnicity, and sex-adjusted HR being 6.0 (95\% CI 3.9-9.1) for the highest CAC quartile versus zero CAC $(\mathrm{p}<0.0001)$. The results for $\mathrm{CHD}$ risk (not shown) were similar. For reference to recommended clinical cutpoints for CAC, 1,3 the age, race/ethnicity, and sex-adjusted HRs (95\% CIs) for CAC scores of $0,1-99,100-399$, and $\geq 400$ were 1.0, 4.7 (95\% CI 2.5-8.7), 11.5 (95\% CI 6.221.5), and 16.1 (95\% CI 8.5-30.8), respectively (not shown in tables).

When put in the same model, CAC was more strongly associated than was IMT with both CVD and CHD (Table 2). The multivariable adjusted HRs (95\% CI) of CVD and CHD per standard deviation increment were $2.1(1.8-2.5)$ and $2.5(2.1-3.1)$, respectively, for $\ln (\mathrm{CAC}+1)$, compared with 1.3 (1.1-1.4) and 1.2 (1.0-1.4) for Z score maximum IMT. Furthermore, the Z statistics were larger and $p$-values were smaller for the CAC association. In contrast, for stroke, only Z score maximum IMT was statistically significant $(\mathrm{p}<0.05)$ with multivariable-adjusted hazard ratio of $1.3(1.1-1.7)$ while the hazard ratio for $\ln (\mathrm{CAC}+1)$ was $1.1(0.8-1.4)$.

A categorical analysis (Table 3) also suggested CAC predicted incident CVD and CHD better than did IMT. For example, the multivariable-adjusted HRs of CHD for the highest quartile 
versus lowest $50^{\text {th }}$ percentile were $8.2(95 \%$ CI $4.5-15.1, \mathrm{p}<0.0001)$ for CAC and 1.7 (95\% CI $1.1-2.7, \mathrm{p}=0.01$ ) for IMT.

In supplemental analysis, we restricted to subjects at intermediate CHD risk, based on a Framingham risk score of $1-2 \%$ per year ( $n=1841$, with 54 CHD events). Among them, the multivariable adjusted HRs (95\% CI) of CHD per standard deviation were 2.4 (1.7-3.3, $\mathrm{p}<0.0001)$ for $\ln (\mathrm{CAC}+1)$ and $1.3(1.0-1.6, \mathrm{p}<0.05)$ for $\mathrm{Z}$ score maximum IMT when both were included in the model. In the same subgroup at intermediate Framingham risk, for CVD (81 events), the multivariable adjusted HRs were $1.8(1.4-2.2, \mathrm{p}<0.0001)$ for $\ln (\mathrm{CAC}+1)$ and $1.4(1.1-1.6, \mathrm{p}=0.001)$ for $\mathrm{Z}$ score maximum IMT.

Figure 1 shows crude rates of incident CVD by 9 joint categories of $Z$ score maximum IMT and CAC. Rates of CVD were between 1-2\% per year for those with (1) a moderate level of CAC and high IMT or (2) a high level of CAC and low IMT. CVD rates were $>2 \%$ per year for those with a high level of CAC and either a moderate or high level of IMT. Those with zero CAC and either low or moderate IMT had almost no events during this follow-up period. Findings for CHD were similar (not shown).

ROC analysis suggested CAC score predicted CVD incidence better than did carotid IMT. With the multiple risk factors in the model for CVD, the area under the curve (AUC) was 0.772 (95\% CI 0.74-0.80). After then adding Z score maximum IMT the AUC was 0.782 (95\% CI 0.75-0.81); after substituting CAC score for IMT was 0.808 (95\% CI 0.78-0.83); and after including both IMT and CAC was 0.811 (0.78-0.84). A similar ROC analysis for CHD produced AUCs of 0.771 (95\% CI 0.74-0.80) for risk factors alone, 0.782 (95\% CI 0.75-0.82) for risk factors plus IMT, 0.823 (95\% CI 0.79-0.85) for risk factors plus CAC, and 0.824 (95\% CI 0.79-0.85) for risk factors plus CAC and IMT.

\section{COMMENT}

This prospective analysis of the MESA cohort initially free of symptomatic CVD found that carotid maximum IMT and CAC, two measures of subclinical atherosclerosis, predicted future CVD events. However, CAC was the better predictor for CHD and total CVD. IMT was a modestly better predictor than CAC of stroke, although there were few stroke events. The associations observed were consistent with those reported by meta-analyses of prospective studies of each subclinical measure of atherosclerosis studied separately. 1,2 They were somewhat inconsistent with a small prospective study in the elderly, in which common carotid IMT was comparable to CAC in predicting CVD and CHD. ${ }^{24}$ It may be that IMT becomes more predictive of CVD in old age, but the smaller sample size of that study also may have limited its ability to show differences between CAC and IMT associations with CVD.

Although previous consensus statements indicated that CAC and IMT are global atherosclerosis measures and either might be used clinically for refinement of CVD risk assessment, ${ }^{4,20}$ our data suggest that in asymptomatic United States adults, CAC may be the better choice over IMT. As judged by proportional hazards modeling and by the AUC, CAC added more to CVD prediction beyond traditional risk factors than did IMT. CAC also was associated more strongly than IMT with CHD within the group of individuals at intermediate risk, for whom a subclinical atherosclerosis assessment may be most appropriate. $1,3,20$ When more CVD events accrue in MESA, we can more thoroughly address the issue of what novel measures (e.g., CAC, IMT, C-reactive protein, and others) might improve CVD risk prediction in intermediate risk patients.

Modestly better prediction of stroke by IMT and clearly better prediction of CHD by CAC likely reflects their different vascular territories. The potential choice between measuring CAC or IMT or neither in preventive cardiology depends upon other considerations as well (e.g., 
differences in radiation exposure, cost, and availability). CAC may be most relevant in the U.S. where CHD is common. If risk of stroke in families with histories of early stroke were a concern, then carotid IMT may be very relevant. Also, in MESA there are substantial ethnic differences in CAC (highest in whites), ${ }^{21}$ and to a lesser degree for IMT (highest in African Americans), ${ }^{22}$ which may impact clinical use.

Strengths of this study include its multiethnic sample, standardized subclinical atherosclerosis assessments and risk factor measurements, and its reliance on symptomatic endpoints to avoid detection bias related to CVD events being diagnosed more readily in subjects with known subclinical atherosclerosis. Limitations include, firstly, the relatively short follow-up and relatively small number of strokes to date. Results could be different for long-term CVD prediction, especially as this population ages and the ratio of strokes to CHD events increases. Secondly, the shapes of distributions differ for IMT and CAC, with many zero values for CAC. However, our analyses using both categorical and continuous measures of IMT and CAC placed them on a more comparable footing. Thirdly, although all endpoints were symptomatic, we included both "hard" CHD (myocardial infarction and CHD death) and "soft" CHD (angina) to provide adequate statistical power. Fourthly, for ethnical reasons we felt compelled to report high CAC and IMT values to participants and refer them to their physicians. More participants were referred for high CAC (17\%) than for high IMT (1\%), which could have affected our findings if participants changed risk factors differentially. Yet, this seems unlikely, as a clinical trial suggested that telling patients their CAC score does not motivate significant health behavior change. 23

In conclusion, although whether and how to employ bio-imaging tests for subclinical atherosclerosis remains a topic of debate, this study found that CAC predicted subsequent CVD events better than does carotid IMT.

\section{Acknowledgements}

The authors thank the other investigators, the staff, and the participants of the MESA Study for their valuable contributions. A full list of participating MESA investigators and institutions can be found at http://www.mesa.nhlbi.org.

Funding/Support: This research was supported by N01-HC-95159 through N01-HC-95169 from the National Heart, Lung, and Blood Institute.

\section{References}

1. Greenland P, Bonow RO, Brundage BH, et al. ACCF/AHA 2007 clinical expert consensus document on coronary artery calcium scoring by computed tomography in global cardiovascular risk assessment and in evaluation of patients with chest pain: a report of the American College of Cardiology Foundation Clinical Expert Consensus Task Force (ACCF/AHA Writing Committee to Update the 2000 Expert Consensus Document on Electron Beam Computed Tomography). Circulation 2007;115:402-426. [PubMed: 17220398]

2. Lorenz MW, Markus HS, Bots ML, Rosvall M, Sitzer M. Prediction of clinical cardiovascular events with carotid intima-media thickness. A systematic review and meta-analyses. Circulation 2007;115:459-467. [PubMed: 17242284]

3. Budoff MJ, Achenbach S, Blumenthal RS, et al. American Heart Association Committee on Cardiovascular Imaging and Intervention; American Heart Association Council on Cardiovascular Radiology and Intervention; American Heart Association Committee on Cardiac Imaging, Council on Clinical Cardiology. Assessment of coronary artery disease by cardiac computed tomography: a scientific statement from the American Heart Association Committee on Cardiovascular Imaging and Intervention, Council on Cardiovascular Radiology and Intervention, and Committee on Cardiac Imaging, Council on Clinical Cardiology. Circulation 2006;114:1761-1791. [PubMed: 17015792] 
4. Naghavi M, Falk E, Hecht HS, et al. for the SHAPE Task Force. From vulnerable plaque to vulnerable patient--Part III: Executive summary of the Screening for Heart Attack Prevention and Education (SHAPE) Task Force report. Am J Cardiol 2006;98(2A):2H-15H.

5. Newman AB, Naydeck BL, Sutton-Tyrrell K, et al. Relationship between coronary artery calcification and other measures of subclinical cardiovascular disease in older adults. Arterioscler Thromb Vasc Biol 2002;22:1674-1679. [PubMed: 12377748]

6. Oei H-HS, Vliegenthart R, Hak AE, et al. The association between coronary calcification assessed by electron beam compute tomography and measures of extracoronary atherosclerosis. The Rotterdam Coronary Calcification Study. J Am Coll Cardiol 2002;39:1745-1751. [PubMed: 12039486]

7. Wagenknecht LE, Langefeld CD, Carr JJ, et al. Race-specific relationships between coronary and carotid artery calcification and carotid intimal medial thickness. Stroke 2004;35:e97-e99. [PubMed: 15073382]

8. Barrett-Connor E, Laughlin GA, Connor C. Coronary artery calcium versus intima-media thickness as a measure of cardiovascular disease among asymptomatic adults (from the Rancho Bernardo Study). Am J Cardiol 2007;99:227-231. [PubMed: 17223423]

9. Arad Y, Spadaro LA, Roth M, et al. Correlations between vascular calcification and atherosclerosis: a comparative electron beam CT study of the coronary and carotid arteries. J Comput Assist Tomogr 1998;22:207-211. [PubMed: 9530380]

10. Wagenknecht LE, Langefeld CD, Carr JJ, et al. Race-specific relationships between coronary and carotid artery calcification and carotid intimal medial thickness. Stroke 2004;35:e97-e99. [PubMed: 15073382]

11. Terry JG, Carr JJ, Tang R, et al. Coronary artery calcium outperforms carotid artery intima-media thickness as a noninvasive index of prevalent coronary artery stenosis. Arterioscler Thromb Vasc Biol 2005;25:1723-1728. [PubMed: 15947237]

12. Brook RD, Bard RL, Patel S, et al. A negative carotid plaque area test is superior to other noninvasive atherosclerosis studies for reducing the likelihood of having underlying significant coronary artery disease. Arterioscler Thromb Vasc Biol 2006;26:656-662. [PubMed: 16357319]

13. van der Meer IM, Bots ML, Hofman A, del Sol AI, van der Kuip DAM, Witteman JCM. Predictive value of noninvasive measures of atherosclerosis for incident myocardial infarction. The Rotterdam Study. Circulation 2004;109:1089-1094. [PubMed: 14993130]

14. Bild DE, Bluemke DA, Burke GL, et al. Multi-Ethnic Study of Atherosclerosis: Objectives and design. Am J Epidemiol 2002;156:871-881. [PubMed: 12397006]

15. Yaghoubi S, Tang W, Wang S, et al. Offline assessment of atherosclerotic coronary calcium from electron beam tomograms. Am J Cardiac Imag 1995;9:231-236.

16. Agatston AS, Janowitz WR, Hildner FJ, Zusmer NR, Viamonte M Jr, Detrano R. Quantification of coronary artery calcium using ultrafast computed tomography. J Am Coll Cardiol 1990;15:827-832. [PubMed: 2407762]

17. Carr JJ, Nelson JC, Wong ND, et al. Calcified coronary artery plaque measurement with cardiac CT in population-based studies: Standardized protocol of Multi-Ethnic Study of Atherosclerosis (MESA) and Coronary Artery Risk Development in Young Adults (CARDIA) Study. Radiology 2005;234:35-43. [PubMed: 15618373]

18. O'Leary DH, Polak JF, Wolfson SK Jr, et al. Use of sonography to evaluate carotid atherosclerosis in the elderly: The Cardiovascular Health Study. Stroke 1991;22:1155-1163. [PubMed: 1926258]

19. The Sixth Report of the Joint National Committee on Prevention, Detection, Evaluation, and Treatment of High Blood Pressure. Arch Intern Med 1997;157:2413-2446. [PubMed: 9385294]

20. Greenland P, Smith SC Jr, Grundy SM. Improving coronary heart disease risk assessment in asymptomatic people: role of traditional risk factors and noninvasive cardiovascular tests. Circulation 2001;104:1863-1867. [PubMed: 11591627]

21. Bild DE, Detrano R, Peterson D, et al. Ethnic differences in coronary calcification: the Multi-Ethnic Study of Atherosclerosis (MESA). Circulation 2005;111:1313-1320. [PubMed: 15769774]

22. Manolio TA, Arnold AM, Post W, et al. Ethnic differences in the relationship of carotid atherosclerosis to coronary calcification: The Multi-Ethnic Study of Atherosclerosis. Atherosclerosis. 2007 [PubMed: 17412347][Epub ahead of print] 
23. O'Malley PG, Feuerstein IM, Taylor AJ. Impact of electron beam tomography, with or without case management, on motivation, behavioral change, and cardiovascular risk profile: A randomized controlled trial. JAMA 2003;289:2215-2223. [PubMed: 12734132]

24. Newman AB, Naydeck BL, Ives DG, Boudreau RM, Sutton-Tyrrell K, O’Leary DH, Kuller LH. Am J Cardiol 2008;101:186-192. [PubMed: 18178404] 


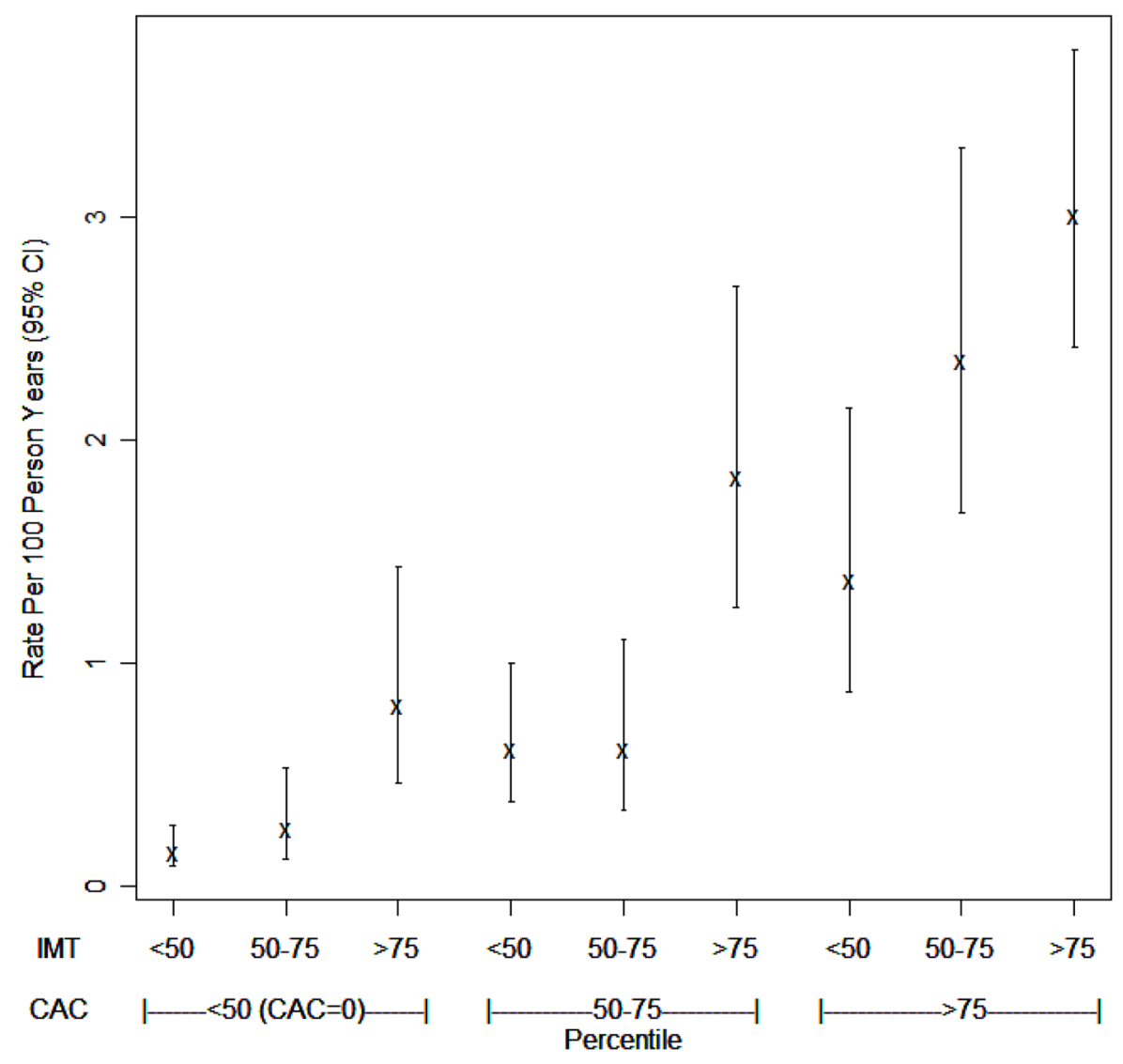

Figure 1.

Unadjusted Rate and 95 Percent Confidence interval of Cardiovascular Disease in Relation to Percentiles of Maximal Carotid Intima-Media Thickness (IMT) or Coronary Artery Calcium (CAC), MESA, 2000-2004 


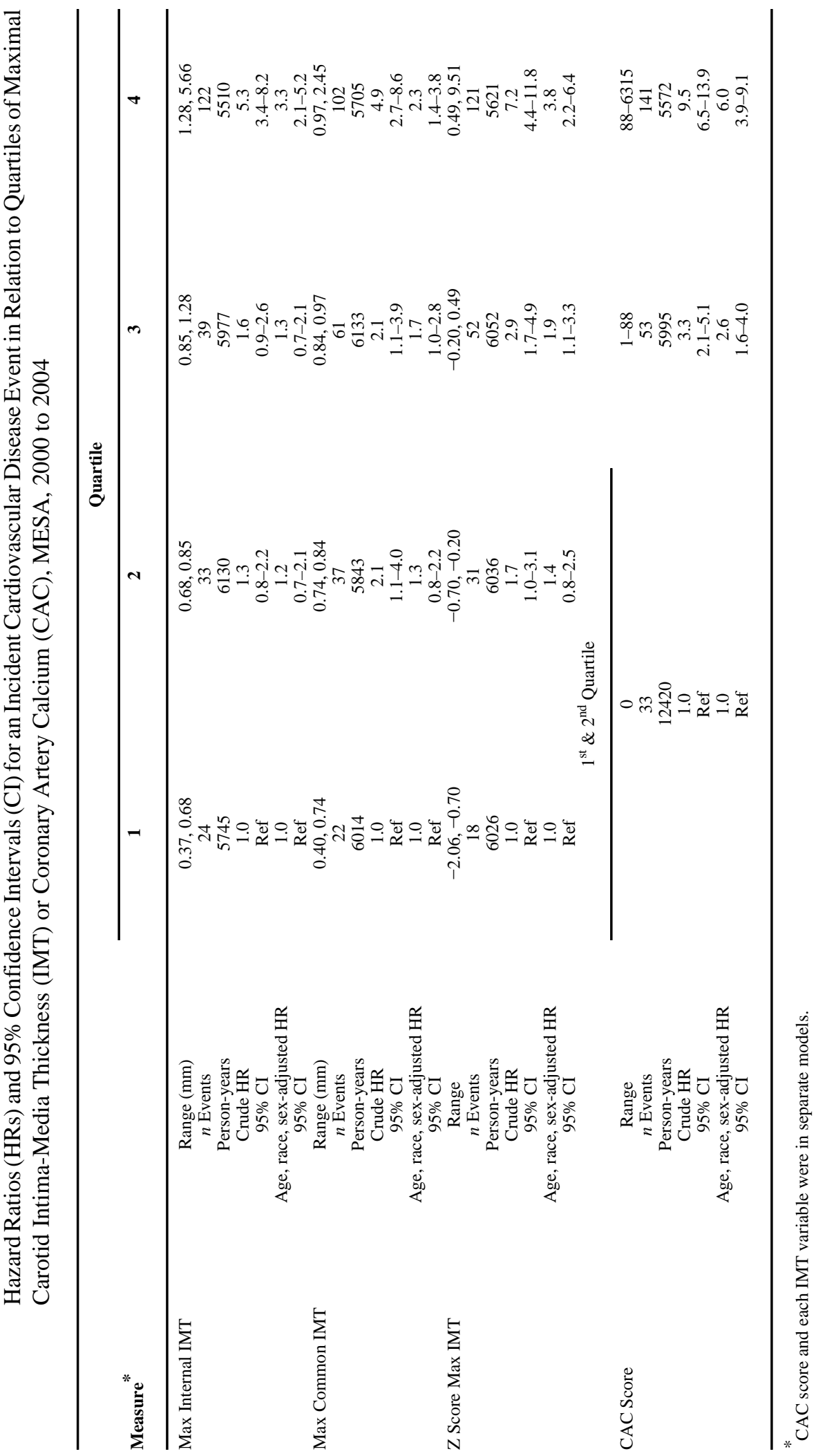


Table 2

Hazard Ratios (HRs) and 95\% Confidence Intervals (CI) for an Incident Cardiovascular Disease (CVD), Coronary Heart Disease (CHD), or Stroke Event in Relation to One Standard Deviation (SD) Increment of Maximal Carotid Intima-Media Thickness (IMT) or Coronary Artery Calcium (CAC), MESA, 2000 to 2004

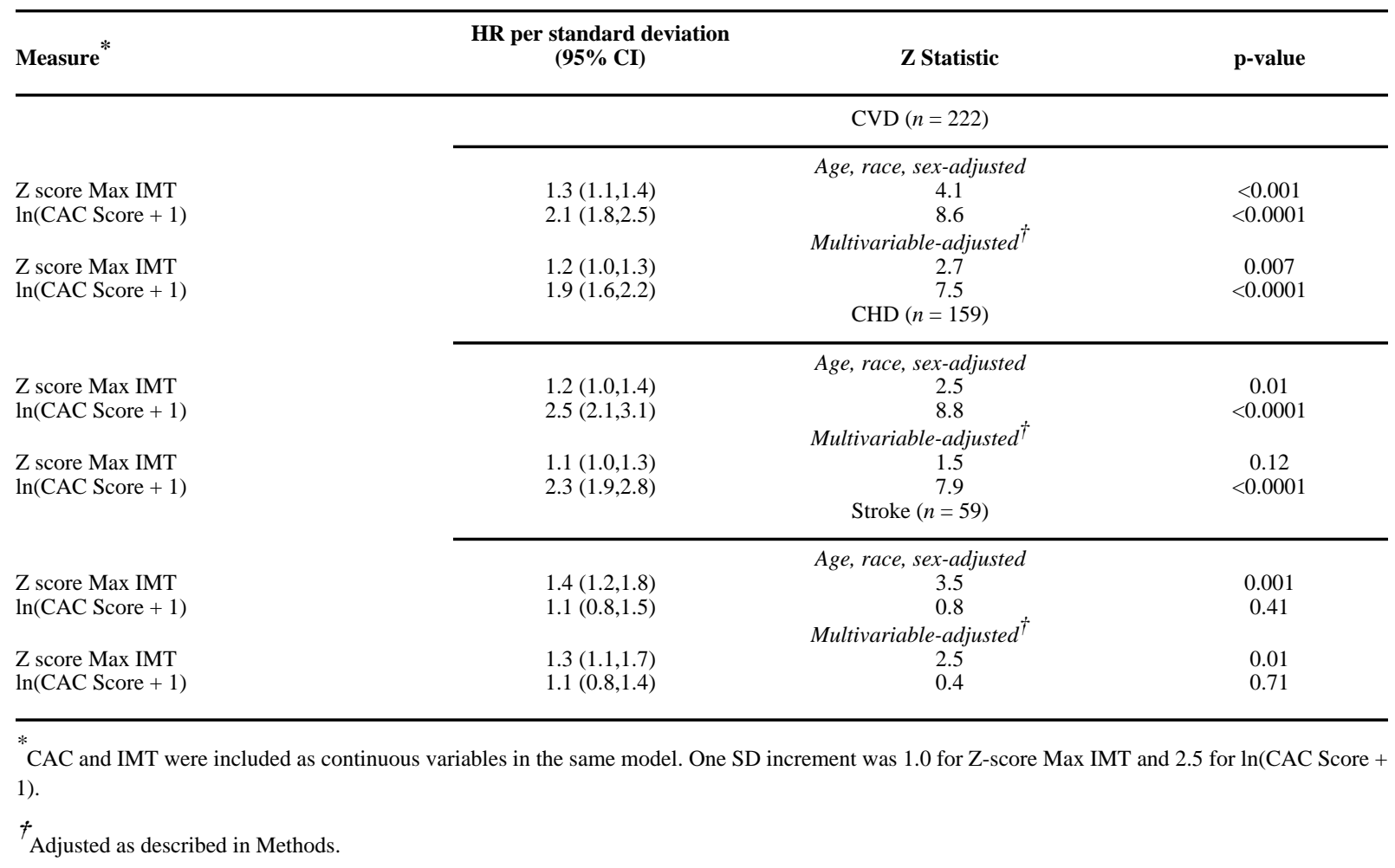


Folsom et al.

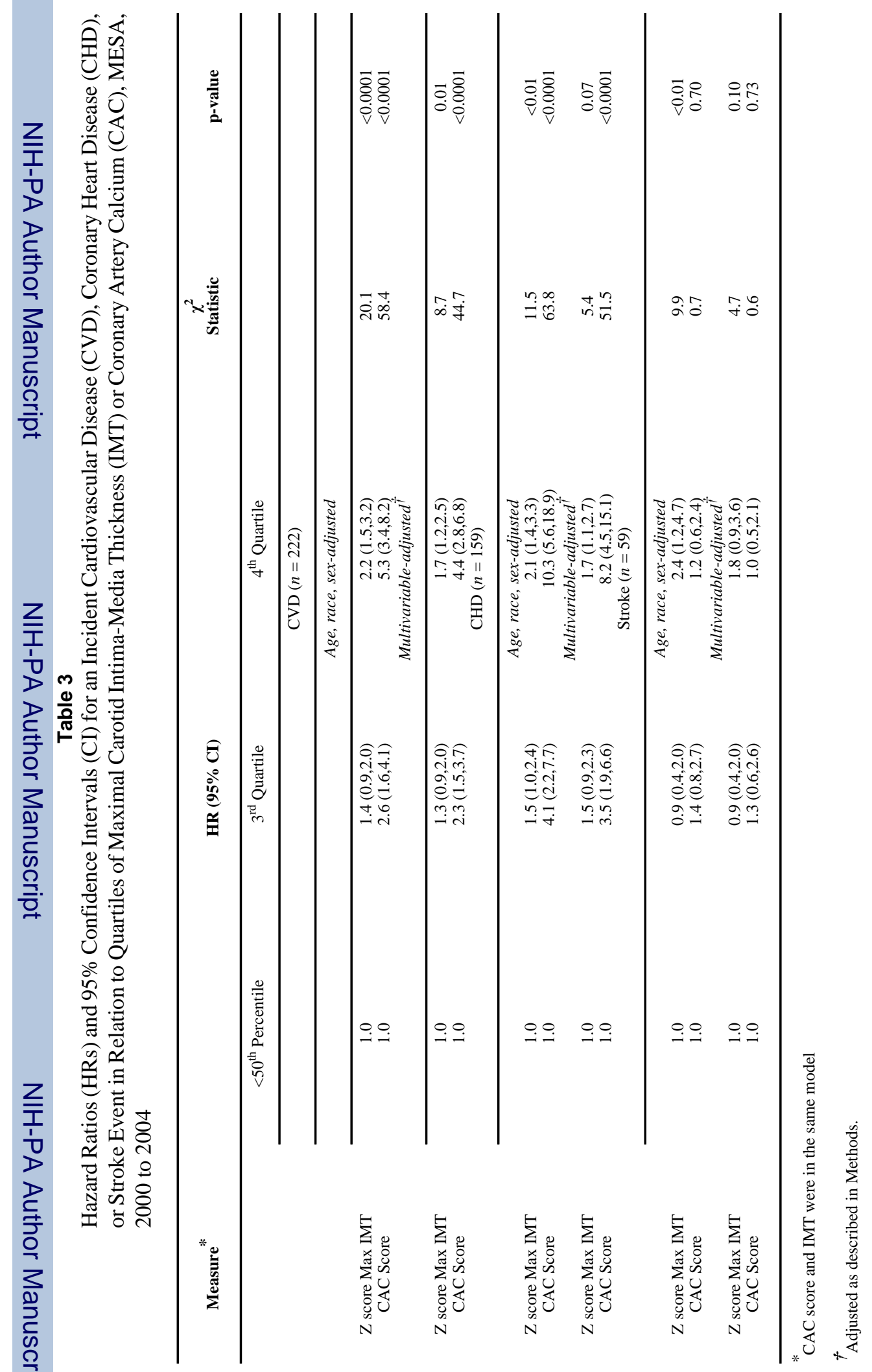

